\title{
Andreev reflection through a ferromagnet-quantum dot-superconductor system with intradot Coulomb correlations
}

\author{
Kacper Bocian and Wojciech Rudziński ${ }^{\mathrm{a}}$ \\ Faculty of Physics, Adam Mickiewicz University, ul. Umultowska 85, 61-614 Poznań, Poland
}

\begin{abstract}
Spin-dependent tunneling through a quantum dot coupled to one ferromagnetic and one superconducting electrodes is studied in the Andreev reflection (AR) regime. Electrical conductance is calculated within the nonequilibrium Green function technique. Effects due to a competition between the Coulomb correlations on the dot and intradot spin-flip processes are considered in the linear transport regime and for different coupling strengths between the dot and the external electrodes. It is shown that when a coherent spin rotation is present on the dot, Coulomb interactions may lead to a significant enhancement of the AR tunneling current and even to the perfect AR transmission. Origin of occurrence of a variety of the multipeak structure of the linear conductance is also discussed.
\end{abstract}

\section{Introduction}

Transport through hybrid structures consisting of quantum point contacts, quantum dots, nanoparticles, carbon nanotubes or other molecules attached via tunnel barriers to external nonmagnetic, ferromagnetic or superconducting leads have been the subject of extensive studies in recent years. It has been shown that the linear conductance due to Andreev reflection (AR) allows to determine spin polarization at the Fermi level of an external ferromagnetic metallic electrode attached to a superconducting point contact [1]. Further advances in nanofabrication techniques have made it possible to make devices working as double quantum dot-based Cooper pair splitters, quantum interferometers with InAs quantum dot bridging elements or tunneling junctions with superconducting electrodes attached to the central island being a self assembled SiGe quantum dot or a carbon $C_{60}$ molecule $[2]$.

On theoretical side, the effects due to competition between superconducting pairing, Coulomb blockade, spin-flip scattering, interaction with boson field and Zeeman effect have been studied in electronic transport through a system consisting of ferromagnet $(\mathrm{F})$, quantum dot (QD) and superconductor $(\mathrm{S})[3-7]$. The Andreev reflection in transport through a QD coupled to one or two ferromagnetic electrodes and a superconductor revealed some interesting properties associated with the matching condition for maximum $\left(4 e^{2} / h\right)$ linear conductance and the magnetoresistance effect $[3,4]$. It has been also predicted that for strong enough spin-flip interaction in the dot, the interplay of spin properties of the AR processes and spin dependent electronic transport leads to a double-peak structure in the linear conductance [4]. Moreover, it is found that when the electron-phonon interactions in the dot are taken into account, new peaks appear on

\footnotetext{
a e-mail: wojrudz@amu.edu.pl
}

both sides of the main AR conductance peak and the splits due to spin-flip scattering appear also in these new satellite peaks [5]. In turn, in [6] the MeserveyTedrow-Fulde effect near the gap-edge energies has been predicted for a quantum dot embedded between normal metallic (nonmagnetic) and superconducting electrodes. The discussion of the latter work revealed also that the on-dot Coulomb interactions impose influence on the in-gap Andreev current leading to the Coulomb satellites in the conductance and can produce a narrow Kondo resonance. Finally, in [7] effects due to Zeeman splitting of the QD level have been analyzed in a hybrid F-QD-S junction in both linear and nonlinear transport regimes and it is found that the net spin polarization and spin accumulation in the dot gives rise to a strong asymmetry of the Andreev reflection double-peak conductance resonance.

In the present paper we investigate features of the Andreev reflection phenomenon in a F-QD-S tunneling junction, by considering interplay between tunneling processes, intradot coherent spin rotation and Coulomb correlations on the dot. Our discussion generalizes theoretical analysis of the AR effect in mesoscopic systems proposed previously in $[4,6]$. The assumption of arbitrary Coulomb correlations extends description of the AR effect to a general case of QD attached to a normal metallic or ferromagnetic external electrode. It is shown that the spin-dependent tunneling in the presence of the competing intradot electron-electron interactions and spin-flip scattering may give rise to new effects modifying the AR transport characteristics. In particular, the conditions leading to a significant enhancement or even to the perfect AR transmission in the hybrid F-QD-S system will be discussed in detail.

In order to calculate the current-voltage characteristics we employ the nonequilibrium Green function technique, and use the equation of motion method. The solutions for the AR current and differential con-

This is an Open Access article distributed under the terms of the Creative Commons Attribution License 2.0, which permits unrestricted use, distribution, and reproduction in any medium, provided the original work is properly cited. 
ductance were obtained within the so called Hubbard I approximation applied to higher-order Green functions.

\section{Model and method}

We consider a single-level QD coupled via tunnel barriers to one ferromagnetic and one superconducting leads. The whole system can be described by Hamiltonian of the general form

$$
H=H_{f}+H_{s}+H_{d}+H_{t} .
$$

The term $H_{f}$ describes the ferromagnetic electrode in the non-interacting quasi-particle approximation, $H_{f}=\sum_{k, \sigma} \varepsilon_{k \sigma} f_{k \sigma}^{+} f_{k \sigma}$, where $\varepsilon_{k \sigma}$ is the single-electron energy for wavevector $\mathrm{k}$ and $\operatorname{spin} \sigma(\sigma=\uparrow, \downarrow)$ in the ferromagnetic electrode, whereas $f_{k \sigma}^{+}$and $f_{k \sigma}$ are the corresponding creation and annihilation operators. The second term is the BCS Hamiltonian of the superconducting lead, $H_{s}=\sum_{p, \sigma} \varepsilon_{p} s_{p \sigma}^{+} s_{p \sigma}+\sum_{p}\left(\Delta^{*} s_{p \uparrow}^{+} s_{-p \downarrow}^{+}+\right.$ $\left.\Delta s_{-p \downarrow} s_{p \uparrow}\right)$, with $\varepsilon_{p}$ denoting the single-particle spectrum in the corresponding normal-metal phase, and $\Delta$ being the parameter describing the superconducting energy gap. The quantum dot is described by the term $H_{d}=\sum_{\sigma} \varepsilon_{d} d_{\sigma}^{+} d_{\sigma}+R\left(d_{\uparrow}^{+} d_{\downarrow}+d_{\downarrow}^{+} d_{\uparrow}\right)+U d_{\uparrow}^{+} d_{\uparrow} d_{\downarrow}^{+} d_{\downarrow}$, where $\varepsilon_{d}$ denotes the energy of the discrete level, $d_{\sigma}^{+}$ and $d_{\sigma}$ are the relevant creation and annihilation operators, respectively, with spin $\sigma(\sigma=\uparrow, \downarrow), R$ is the spin-flip coupling parameter (e.g. due to spin-orbit interaction or magnetic field normal to the quantization axis), while $U$ is the on-dot Coulomb repulsion between opposite spin electrons. Finally, the tunnelling term in Eq.(1) takes the form $H_{t}=\sum_{k, \sigma} T_{k \sigma}^{f} f_{k \sigma}^{+} d_{\sigma}+$ $\sum_{p, \sigma} T_{p \sigma}^{s} s_{p \sigma}^{+} d_{\sigma}+$ h.c., where $T_{k \sigma}^{f}\left(T_{p \sigma}^{s}\right)$ is the tunneling amplitude between the ferromagnetic (superconducting) lead and the QD, and h.c. stands for the hermitian conjugate terms.

As stated above, the incident electrons with upspin and down-spin from the $\mathrm{F}$ lead are assumed to tunnel coherently onto the discrete level split by the intradot spin flips. This tunneling of electrons into a superposition of spin-up and spin-down states may be introduced by using spin rotation canonical transformation $[8] c_{\uparrow(\downarrow)}=\frac{1}{\sqrt{2}}\left(d_{\uparrow} \mp d_{\downarrow}\right)$, where $c_{\uparrow}$ and $c_{\downarrow}$ are new Fermi operators. In terms of above new Fermi operators, the Hamiltonian $H_{d}$ is rewritten as $H_{d}=$ $\sum_{\sigma} \varepsilon_{d \sigma} c_{\sigma}^{+} c_{\sigma}+U c_{\uparrow}^{+} c_{\uparrow} c_{\downarrow}^{+} c_{\downarrow}$, with $\varepsilon_{d \uparrow(\downarrow)}=\varepsilon_{d} \mp R$, whereas the tunneling Hamiltonian $H_{t}$ takes the form $H_{t}=$ $\frac{1}{\sqrt{2}} \sum_{k, \sigma}\left(T_{k \uparrow} f_{k \uparrow}^{+}+(-1)^{\delta_{\uparrow \sigma}} T_{k \downarrow} f_{k \downarrow}^{+}\right) d_{\sigma}+\frac{1}{\sqrt{2}} \sum_{k, \sigma}\left(T_{k \uparrow} s_{k \uparrow}^{+}+\right.$ $\left.(-1)^{\delta_{\uparrow \sigma}} T_{k \downarrow} s_{k \downarrow}^{+}\right) d_{\sigma}+$ h.c.

Making use of the nonequilibrium Green-function technique in the $4 \times 4 \mathrm{Nambu}$ representation [9], one can calculate the current due to Andreev reflection from the formula $[4,9]$ :

$J=\frac{e}{h} \int d \omega\left[f_{l}(\omega-e V)-f_{l}(\omega+e V)\right] \Sigma_{i=1,3}^{j=2,4} G_{i j}^{r}\left(\boldsymbol{\Gamma}_{f} \mathbf{G}^{a} \boldsymbol{\Gamma}_{f}\right)_{j i}$,

where $f_{l}(\omega)$ stands for the Fermi-Dirac distribution function in the left (ferromagnetic) lead, and $\mathbf{G}^{r(a)}$ is the retarded (advanced) Green function of the QD.
Apart from this, $\boldsymbol{\Gamma}_{f}$ in Eq.(2) is the coupling matrix between the QD and ferromagnetic lead, which is determined by the spin-up and spin-down coupling strengths defined as $\Gamma_{f \uparrow(\downarrow)}=\Gamma_{f 0}(1 \pm P)$, where $P$ is the corresponding spin polarization factor and $\Gamma_{f 0}$ is a coupling constant. Both $\boldsymbol{\Gamma}_{f}$ and $\mathbf{G}^{r(a)}$ are matrices in the Nambu space.

With these definitions, the matching condition of the Fermi velocity for perfect Andreev reflection can be written as $\Gamma_{f \uparrow} \Gamma_{f \downarrow}=\Gamma_{s 0}^{2}$. Here, $\Gamma_{s 0}$ describes the tunneling coupling strength between the QD and the superconducting lead.

\section{Numerical results}

In the following we shall discuss features of the AR conductance at zero temperature, $T=0$, for the hybrid F-QD-S system in the linear response regime, $V \approx 0$. Energy is measured from the Fermi level at equilibrium, and for the AR current calculations the energy gap of the superconducting lead is assumed to be $\Delta \rightarrow \infty$. The spin polarization $P$ is taken as $P=0.3$. All energy-dependent parameters are given in unit $e V$.

In Fig. 1 we show the linear AR conductance versus energy level $\varepsilon_{d}$ of the QD, calculated for a fixed $\Gamma_{s 0}$, $\Gamma_{s 0}=0.1$, for indicated values of the coupling strength $\Gamma_{f 0}$, and for two values of the Hubbard parameter $U$ : $U=0$ (solid lines in Fig. 1) and $U=0.15$ (dashed lines in Fig. 1). To ephasize the features due to Coulomb correlations in the dot, the intradot spin-flip processes have been excluded in Fig. 1 assuming $R=0$. If $U=0$, the AR conductance exhibits one resonance peak at $\varepsilon_{d}=0$. As one can see in Fig. 1a, the conductance is relatively small for $\Gamma_{f 0}<\Gamma_{s 0}$. When the coupling strength $\Gamma_{f 0}$ increases, then for the specific case of $\Gamma_{f 0}=\Gamma_{s 0}$, the perfect AR tunneling at $\varepsilon_{d}=0$ occurs. This property is shown by the solid line in Fig. $1 \mathrm{~b}$. Further increasing of the parameter $\Gamma_{f 0}$ (see Fig. 1c) reduces the Andreev reflection and consequently the height of the AR resonance peak is suppressed. For non-zero Coulomb interactions on the dot the linear conductance has two resonances located at energies $\varepsilon_{d}=0$ and $\varepsilon_{d}=-U$, which means that due to the AR processes the dot may be occupied by two carriers with opposite spins.

In Fig. 2 the AR linear conductance is shown for selected values of the spin flip rate $R$ and for a fixed Hubbard parameter $U=\Gamma_{s 0} / 2$, in situation when the coupling strength between the dot and the external leads is given by the relation $\Gamma_{f 0}<\Gamma_{s 0}$. For comparison the conductance resonances obtained for vanishing on-dot repulsion $(U=0)$ are also displayed (see insets in Fig. 2). As stated earlier in [4], if $U=0$, then for a weak spin-flip scattering strength determined by $R \subset\left(0, \Gamma_{s 0} / 2\right)$, the AR conductance exhibits a singlepeak resonance at $\varepsilon_{d}=0$ and with increasing $R$ it is gradually enhanced until the maximum $G_{m}=4 e^{2} / h$ at $R=\Gamma_{s 0} / 2$ (see insets in Fig.2a and Fig.2b). Such a behavior is due to the fact that for $R \leq \Gamma_{s 0} / 2$ the resonant broadening $\Gamma=\Gamma_{f 0}+\Gamma_{s 0}$ gives rise to overlap of the spin-coherent split levels $\varepsilon_{d}= \pm R$ and conse- 
quently to an enhanced transmission of the AR current through the F-QD tunneling juction. For stronger splin-flip scatterings $R>\Gamma_{s 0} / 2$ first the amplitude of the resonant peak reduces quickly and then develops into a double peak resonance with two peaks at $\varepsilon_{d}= \pm R$ (inset in Fig.2c).

It is clearly seen that in the presence of on-dot Coulomb correlations the single-peak resonance develops into the three-peak or the four-peak structure. In the considered case of $\Gamma_{f 0}<\Gamma_{s 0}$ the latter may be accompanied by an enhancement (up to $3.5 e^{2} / h$ ) of the linear conductance if $R \leq U$. In fact, the conductance now has two resonances and due to spin-flip scattering each of them is split into two additional peaks at energies $\varepsilon_{d}= \pm R$ and $\varepsilon_{d}=-U \pm R$. In the case of $R<U$ (Fig.2a) one observes only three peaks since for a small enough $R$ the linewidth of the split levels $\varepsilon_{d}+R$ and $\varepsilon_{d}+U-R$ overlap effectively giving rise to the middle conductance peak at $\varepsilon_{d}=-U / 2$. On the other hand, the prominent external left and right satellite resonances in Fig.2a indicate on increased AR current through the spin-split levels $\varepsilon_{d}+U+R$ and $\varepsilon_{d}-R$. In this particular case the effective overlap of the discrete levels is determined by the specific relation between $R, U$ and $\Gamma$, so that the in-gap AR enhanced transmission is observed at resonances separated in energy scale by $U+2 R$.

Fig.2b shows that Coulomb correlations slightly suppress the perfect AR observed for $U=0$ and $R=$ $\Gamma_{s 0} / 2$. Moreover, when $U=R$, instead of the middle maximum in fig.2a, now two resonances separated by a minimum at $\varepsilon_{d}=-U / 2$ appear. Also, by contrast to the situation displayed in Fig.2a, if $U=R$ the AR transmission is now rather more enhanced through the energy levels $\varepsilon_{d}+R$ and $\varepsilon_{d}+U-R$.

Finally, when $R>U$, the conductance maxima are reduced due to the intradot spin-flip scattering processes, however the two-peak resonance observed for vanishing Coulomb correlations in the dot and large enough $R$, now develops into the four-peak resonance, which is symmetrical around $\varepsilon_{d}=-U / 2$ (see Fig.2c). The two narrow internal peaks correspond to AR transmission through the levels $\varepsilon_{d}+R$ and $\varepsilon_{d}+U-R$, whereas the broad external ones come from tunneling through the levels $\varepsilon_{d}-R$ and $\varepsilon_{d}+U+R$.

As discussed in [4], the conditions for the perfect AR in the F-QD-S system depend on the symmetry of the coupling strength between the dot and the external leads. For $R=U=0$ only in the case of $\Gamma_{f 0}=\Gamma_{s 0}$ the linear conductance reaches the maximum value $4 e^{2} / h$. If coherent spin rotation on the dot is switched on $(R>0)$ the matching condition may be filled for asymmetrical coupling between the dot and the leads, $\Gamma_{f 0}<\Gamma_{s 0}$, at $R=\Gamma_{s 0} / 2$.

Fig. 3 shows the situations for which one may observe the highest conductance resonance peaks, when different relations between spin-flip processes and Coulomb interactions on the dot are taken into account. For a weak coupling between the dot and the F electrode, $\Gamma_{f 0}<\Gamma_{s 0}$ and small $R$, the Coulomb repulsion on the dot gives rise to an enhancement of the ingap AR current (see Fig.3a and also Fig.2a and b). The amplitude of the conductance peak reaches then

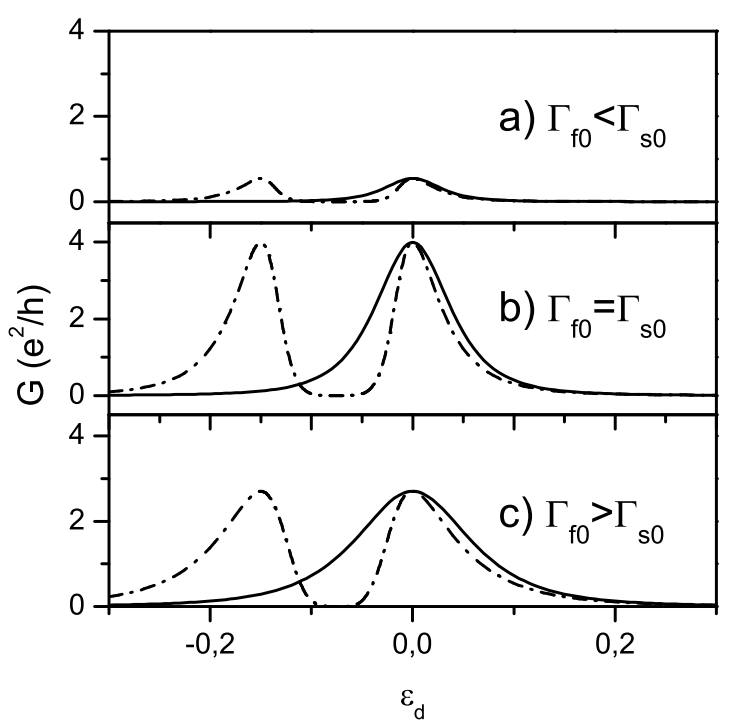

Fig. 1. AR linear conductance versus energy of the quantum dot discrete level, $\varepsilon_{d}$, calculated for indicated values of the Hubbard parameter $U=0$ (solid lines), $U=0.15$ (dashed lines) and $R=0$. The other parameters are: $T=0, P=0.3, \Gamma_{s 0}=0.1, \Gamma_{f 0}=0.02(\mathrm{a}), \Gamma_{f 0}=0.1$ (b), and $\Gamma_{f 0}=0.2(\mathrm{c})$.

approximately $3.5 e^{2} / h$ and remains constant with increasing $U$. The behavior of the $G$ characteristics in the case of symmetrical coupling between the dot and the leads, $\Gamma_{f 0}=\Gamma_{s 0}$, is different. As seen in Fig. $3 \mathrm{~b}$, for the specific relation $U=2 R=\Gamma_{s 0}$, the conductance reaches the maximum value $G_{m}=4 e^{2} / h$ at energy $\varepsilon_{d}=-U / 2$, which means that the matching condition for the Fermi velocity is met. Note that the displayed in Fig.3b characteristics $G\left(\varepsilon_{d}\right)$ represents a general property found in our numerical analysis, that allows to control the matching condition of the Fermi velocity in the F-QD-S system with a fixed $U$ and symmetrical coupling strength between the dot and the leads. Namely, it is found that by manipulating the coherent spin rotation in the dot, the perfect, $U$-dependent AR phenomenon appears always if $R=U / 2$.

In the case of $\Gamma_{f 0}>\Gamma_{s 0}$ (Fig.3c), the conductance maxima become reduced, however for a strong enough intradot spin-flip scattering accompanied by increasing on-dot Coulomb repulsion, one may observe an enhancement of the linear conductance peak at $\varepsilon_{d}=-U / 2$.

\section{Summary and conclusions}

Using the non-equilibrium Green function approach we have considered spin-polarized transport, associated with Andreev reflection, through a single-level interacting quantum dot coupled to one ferromagnetic and one superconducting leads. We have shown that the competition between broadening of the discrete dot's level, coherent intradot spin rotation and on-dot Coulomb repulsion between opposite spin electrons may lead to a multi-peak resonances in the linear AR 


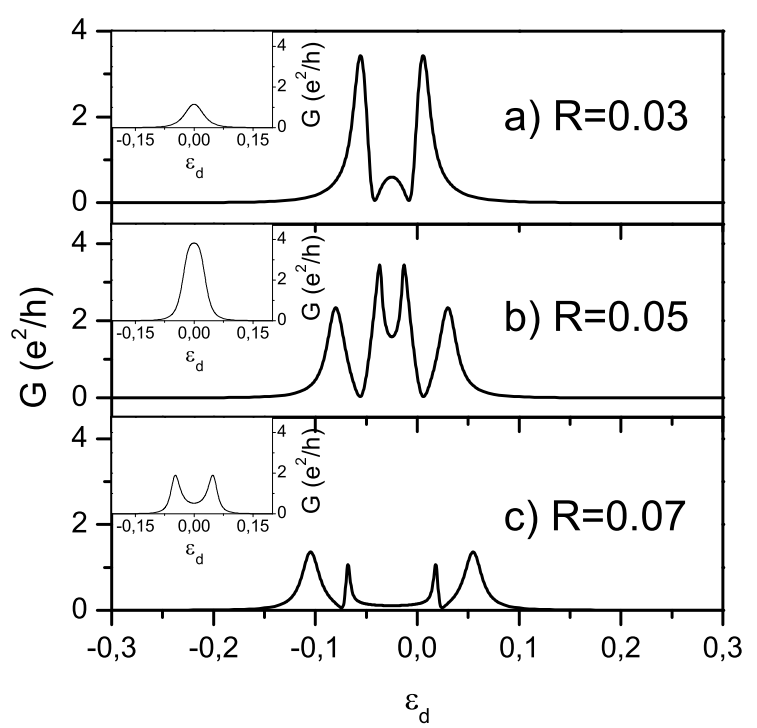

Fig. 2. AR linear conductance versus energy of the quantum dot discrete level, $\varepsilon_{d}$, calculated for the case of $\Gamma_{f 0}<$ $\Gamma_{s 0}$ and for the selected spin-flip rate $R$. The Hubbard parameter is taken as $U=0.05$. The insets show the corresponding resonances for $U=0$. The other parameters are: $T=0, P=0.3, \Gamma_{f 0}=0.02$ and $\Gamma_{s 0}=0.1$.

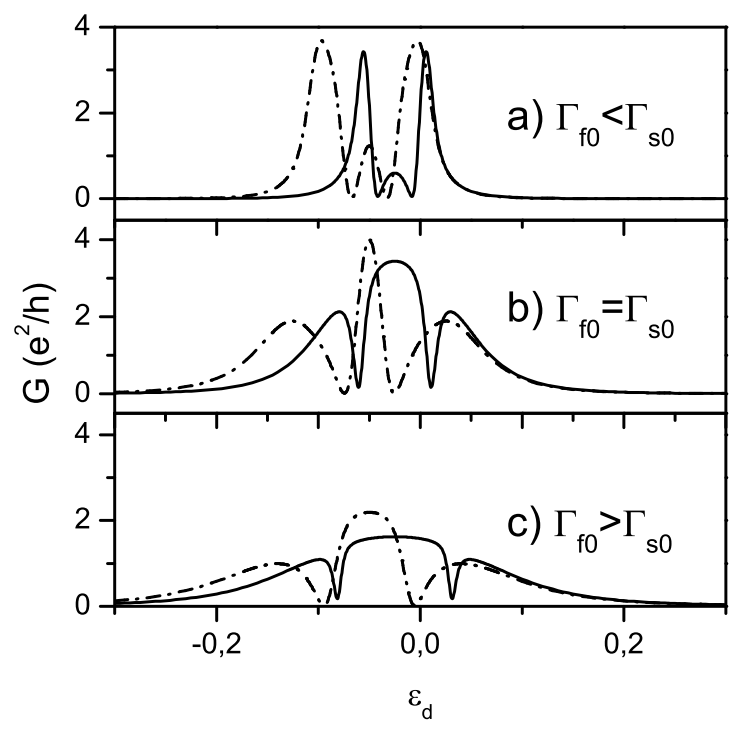

Fig. 3. AR linear conductance versus energy of the quantum dot discrete level, $\varepsilon_{d}$, calculated for the Hubbard parameter $U=0.05$ (solid lines), $U=0.1$ (dashed lines) and spin-flip rate $R=0.03$ (a), $R=0.05$ (b) and $R=0.07$ (c). The other parameters are as in Fig. 1.

conductance. It is found that if $\Gamma_{f 0}<\Gamma_{s 0}$, then for a fixed spin-flip rate $R \leq \Gamma_{s 0} / 2$, a significant enhancement of the linear conductance with increasing $U$ may occur, leading to three-peak or four-peak resonances. In turn, for the case of $\Gamma_{f 0} \geq \Gamma_{s 0}$ the AR transmission may be enhanced only at the dot discrete level energy $\varepsilon_{d}=-U / 2$ if $R \geq \Gamma_{s 0} / 2$.
Moreover, it is shown, that the matching condition of the Fermi velocity, $\Gamma_{f \uparrow} \Gamma_{f \downarrow}=\Gamma_{s 0}^{2}$, for the considered $F-Q D-S$ system is filled best in two cases. First, a perfect $\mathrm{AR}$ occurs if $\Gamma_{f 0}<\Gamma_{s 0}, U=0$, and $R=\Gamma_{s 0} / 2$. The latter is consistent with the previous result obtained for AR tunneling through a spinsplit discrete level due to spin-flip rotation on a noninteracting $(U=0)$ quantum dot [4]. In our approach the above result has been reproduced by using the defined in Section 2 spin rotation canonical transformation applied to the model (1).

Second, we have formulated a new matching condition for the perfect Andreev reflection in case of arbitrary Coulomb correlations on the dot. The maximum linear conductance, $G_{m}=4 e^{2} / h$, may be observed for symmetrical coupling strength between the dot and external electrodes, $\Gamma_{f 0}=\Gamma_{s 0}$, with intradot spin-flip rate $R=\Gamma_{s 0} / 2$ and the Hubbard parameter $U=\Gamma_{s 0}$.

\section{Acknowledgements}

This work was supported by the Polish Ministry of Science and Higher Education as a research project in the years 2010-2013.

\section{References}

1. R. J. Soulen, J. M. Byers, M. S. Osofsky, N. Nadgorny, T. Ambrose, T. Ambrose, S. F. Cheng, P. R. Broussard, C. T. Tanaka, J. Nowak, J. S. Moodera, A. Barry, J. M. D. Coey, Science 282, 85 (1998).

2. S. De Franceschi, L. Kouwenhoven, Ch. Schönenberger, W. Wernsdorfer, Nature Nanotechnology 5, 703 (2010).

3. Y. Zhu, Q. Sun, T. Lin, Phys. Rev. B 65, 024516 (2001).

4. X. Cao, Y. Shi, X. Song, S. Zhou, Phys. Rev. B 70, 235341 (2004).

5. P. Zhang, Y. Li, J. Phys.: Condens Matter 21, 095602 (2009).

6. T. Domański, A. Donabidowicz, K. I. Wysokiński, Phys. Rev. B 78, 144515 (2008).

7. K. Bocian, W. Rudziński, J. Barnaś, Acta Physica Polonica 121, 1201 (2012).

8. H. Song, S. Zhou, Physics Letters A 372, 6773 (2008).

9. Z.Y. Zeng, F. Claro, B. Li, cond-mat/0110502. 